Lymphoma

\title{
CXCR4 in Waldenström's Macroglobulinema: chances and challenges
}

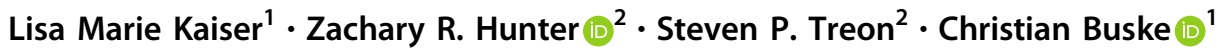

Received: 13 August 2020 / Revised: 9 October 2020 / Accepted: 17 November 2020 / Published online: 3 December 2020

(c) The Author(s) 2020. This article is published with open access

\begin{abstract}
It is one of the major aims in cancer research to improve our understanding of the underlying mechanisms which initiate and maintain tumor growth and to translate these findings into novel clinical diagnostic and therapeutic concepts with the ultimate goal to improve patient care. One of the greater success stories in this respect has been Waldenström's Macroglobulinemia (WM), which is an incurable B-cell neoplasm characterized by serum monoclonal immunoglobulin M (IgM) and clonal lymphoplasmacytic cells infiltrating the bone marrow. Recent years have succeeded to describe the molecular landscape of WM in detail, highlighting two recurrently mutated genes, the MYD88 and the CXCR4 genes: MYD88 with an almost constant and recurrent point mutation present in over $90 \%$ of patients and CXCR4 with over 40 different mutations in the coding region affecting up to $40 \%$ of patients. Intriguingly, both mutations are activating mutations leading in the case of CXCR4 to an indelible activation and perpetual signaling of the chemokine receptor. These data have shed light on the essential role of CXCR4 in this disease and have paved the way to use these findings for predicting treatment response to the Bruton tyrosine kinase (BTK) inhibitor ibrutinib and novel therapeutic approaches in WM, which might be transferable to other related CXCR4 positive diseases. Well known for its central role in cancer progression and distribution, CXCR4 is highlighted in this review with regard to its biology, prognostic and predictive relevance and therapeutic implications in WM.
\end{abstract}

\section{CXCR4-a key member of the chemokine receptor family in hematopoiesis and cancerogenesis}

\section{Structure and ligands}

C-X-C chemokine receptor type 4 (CXCR4), characterized by one amino acid between the two cysteine residues, also named as cluster of differentiation 184 (CD184), is a classical G protein coupled receptor (GPCRs) with its encoded nucleotide sequence located on chromosome 2 [1]. It belongs to the Rhodopsin-like class A of GPCRs, which are membrane proteins of a superfamily that transmit signals from the outside of the cell to activate a plethora of inner molecular pathways. CXCR4 acts as a conventional

Christian Buske

christian.buske@uni-ulm.de

1 Institute of Experimental Cancer Research, CCC and University Hospital Ulm, Germany, 89081 Ulm, Germany

2 Bing Center for Waldenström's Macroglobulinemia, Dana-Farber Cancer Institute, Harvard Medical School, Boston, MA, USA chemokine receptor. Chemokine receptors are organized into four different groups, depending on the chemokine they bind to and the count and arrangement of the $\mathrm{N}$-terminal cysteine residues i.e. CXC, CX3C, CC, and XC [2]. The surface receptor CXCR4 is ubiquitously expressed and evolutionary conserved with a similarity of $89 \%$ between human and mouse orthologues. The surface protein CXCR4 comprising 352 amino acids consists of seven transmembrane helices coiling through the cell membrane of which helix VII is distinctive for CXCR4 compared to other GPCRs. Its uniqueness allows a chemical bond between helix VII and the N-terminal region that belongs to a complex, enabling the binding to CXCL12, the natural ligand of CXCR4 [3].

CXCL12 (stromal cell-derived factor-1, SDF-1), is the natural binding partner of CXCR4 and belongs to a large family of chemokines. The molecular structure of CXCL12 is characterized by a three-stranded $\beta$-sheet that is packed against an $\alpha$-helix [4]. It is ubiquitously expressed in all kinds of tissue with six reported isoforms in human. The chemokine is highly conserved between mouse and human (>92\%), suggesting an important role in evolution and has been mapped to chromosome 10q [5]. Within the hematological system, CXCL12 is secreted by stromal cells in the perivascular 
region, including endothelial cells and mesenchymal progenitors comprising a distinct niche that support HSCs [6]. When genetically deleted, mice have a hypocellular marrow characterized by deficient B-lymphopoiesis and myelopoiesis and abnormal neuronal and cardiovascular development with lethal outcome [7].

CXCL12 binding to CXCR4 initiates a wide network of signal transduction by activating subunit $\mathrm{G} \alpha$ of the heterotrimeric G protein. By switching from GDP to GTP the $\alpha$ unit dissociates from the trimeric structure and binds to GDP. Both, the GTP $\alpha$ and the G $\beta / G \gamma$ dimer interact with different effector proteins and initiate various intracellular signaling cascades. The $\mathrm{G} \alpha$ family acts via PLC, to hydrolyze PIP2 and generates two-second messengers, IP3 and DAG. IP3 and DAG mobilize $\mathrm{Ca} 2+$ from intracellular stores and are able to activate a number of protein kinases, including PKC, controlling migration and proliferation. Further stimulation leads to the activation of mitogenactivated protein kinase (MAPK) originally called ERK (Extracellular signal-regulated kinases) as well as small GTPases RAS and RHO which are involved in chemotaxis and cell cycle regulation. The main axis PI3K is linked to lymphocyte trafficking, chemotaxis, and cell survival via AKT but can also result in the phosphorylation of several adhesion regulators such as proline-rich kinase-2 (Pyk-2), Crk-associated substrate (CRK), focal adhesion kinase (FAK), and paxillin. The PI3K-AKT-NF- $\mathrm{KB}$ axis and also the MEK1/2 and ERK 1/2 pathways are involved in activation and phosphorylation of cellular proteins and transcriptions factors to regulate genes responsible for proliferation, migration, and stemness.

The signaling is tightly regulated and stopped by internalization processes and lysosomal degradation via arrestin which binds to the phosphorylated receptor and initiates degradation [8] (Fig. 1).

Despite CXCL12, other ligands of CXCR4 have been identified, namely ubiquitin [9], the anti-HIV-1 chemokine vMIP-II [10], the HIV protein gp120 [11] and more recently the endogenous CXCR4 antagonist, EPI-X4, an active serum albumin fragment [12].

\section{Functional Role in normal and malignant tissue}

Its crucial role as factor in directed migration and leukocyte trafficking in the immune system of higher organisms as well as homing of stem and progenitor cells has drawn key attention to CXCR4 in the recent years. The role of CXCR4 starts during earliest stages of development at the 4-cell stage in blastocyst formation, where CXCR4 is expressed and gradually increases in expression during embryo development. This increase of CXCR4 has been shown to have a direct effect on the invasion capability and successful implantation of the human blastocyst [13].

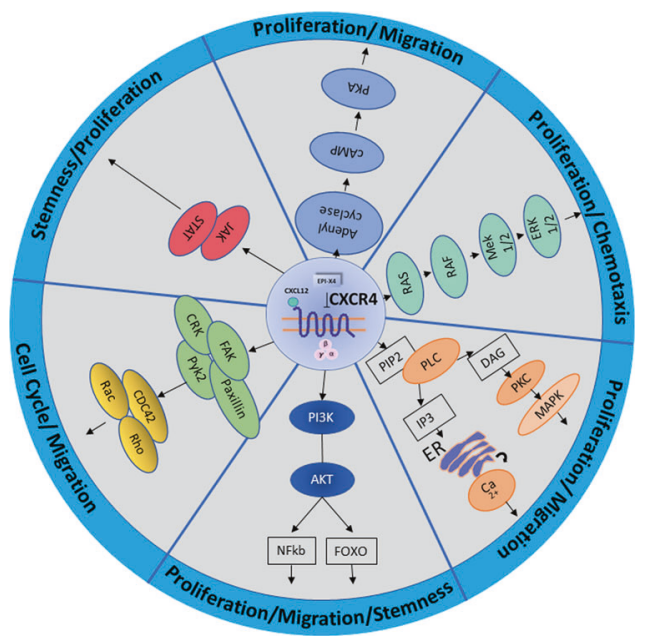

Fig. 1 Schematic overview of CXCR4 signaling. Pathways involved in CXCR4 CXCL12/CXCR4 signaling pathways. AKT protein kinase B, PI3K phosphatidylinositol 3-kinase, NFkB nuclear factor kappalight-chain-enhancer of activated B cells, FOXO forkhead box, FAK focal adhesion kinase, Pyk-2 proline-rich kinase-2, CRK Adapter molecule crk, Rho Ras homolog gene family, Rac Ras-related C3 botulinum toxin substrate, CDC42 cell division control protein 42 homolog, JAK janus kinase, STATsignal transducer and activator of transcription, cAMP cyclic adenosine monophosphate, PKA protein kinase A, Ras rat sarcoma protein family, RAF proto-oncogene serine/ threonine-protein kinase, MEK dual specificity mitogen-activated protein kinase, ERK extracellular-signal-regulated kinase, PIP2 phosphatidylinositol bisphosphate, PLC phospholipase C, PKC protein kinase $\mathrm{C}, \mathrm{IP}_{3}$ inositol trisphosphate, DAG diacylglycerol, ER endoplasmic reticulum, MAPK mitogen-activated protein kinase, $\mathrm{Ca}^{2+}$ Calcium ions, CXCR4 C-X-C chemokine receptor type 4, CXCL12 $\mathrm{C}-\mathrm{X}-\mathrm{C}$ chemokine ligand 12 .

Necessity of CXCR4 for embryonic viability has been shown in mice, as knock-out mice are embryonic lethal with defects in vascular development, hematopoiesis, and cardiogenesis [14]. In later development stages it is involved in organ formation with concise tissue-specific functions for example as leading signal for axon solicitation in neural tissue [15]. Within the hematopoietic system CXCR4 is expressed on most hematopoietic cell types including lymphocytes, stromal fibroblasts, endothelial and epithelial cells as well as hematopoietic stem cells and cancer cells [16]. In the adult BM CXCR4 controls stem cell retention in the BM and its disruption leads to a release of the HSC pool with the ability to restore normal blood cell population or to maintain homeostasis with enhanced release on demand during infections and injuries. Recently, CXCR4 signaling has been found to play a fundamental role in restoring the HSC pool and reconditioning the microenvironment after stem cell transplantation via upregulating the antiapoptotic protein Survivin [17]. These aspects of the role of CXCR4 have been described in more detail in excellent reviews $[15,18]$

CXCR4 has been associated with cancerogenesis for a long time: elevated CXCR4 expression levels have been 
portrayed in numerous cancers with relation to poor prognosis and therapy resistance [19]. Upregulation was linked to several factors, such as hypoxia-inducible factor-1 (HIF-1) [20] as well as several growths factors such as fibroblast growth factor FGF [21], vascular endothelial growth factor (VEGF) [20] and hepatocyte growth factor (HGF) [22]. Also transcription factors like nuclear respiratory factor-1 positively influence CXCR4 expression [23] whereas Yin-Yang 1 (YY1) negatively regulates the promoter activity of the receptor [24]. Furthermore, the macrophage migration inhibitory factor MIF has been associated with tumor aggressiveness and metastatic spread. Upon binding to CXCR4, the MIF/CXCR4 complex is involved in regulation of endothelial progenitor cell migration, metastasis, cancer growth [25] and homing to tumors [26]. However, the implication of CXCR4 with tumorigenesis relies largely on expression studies and functional assays proving direct oncogenicity of CXCR4 in solid cancers is rare.

In hematological malignancies high expression of CXCR4 is observed in several entities ranging from acute leukemias such as acute lymphoblastic leukemia (ALL) and acute myeloid leukemia (AML) to a variety of malignant lymphomas including chronic lymphocytic leukemia (CLL), diffuse large B cell lymphoma (DLBCL), follicular lymphoma (FL), marginal zone lymphoma (MZL), multiple myeloma (MM), hairy cell leukemia (HCL) and mantle cell lymphoma (MCL) [27-32]. Furthermore, detection of the phosphorylated form of CXCR4, pCXCR4, is an indicator for poor survival in adult patients with B-ALL [33]. Furthermore, high expression of CXCR4 is a hallmark of CLL cells compared to normal B cells [34]. Ghobrial et al. saw a significant increase in the expression of CXCR4 in B-CLL cells from patients with RAI stage IV compared with stage 0 [35]. A large multi-center study described CD49d as an independent prognostic marker in CLL and suggested that CD49d and CXCR4 may be up regulated in a co-ordinated fashion and linked to poor prognosis prediction [36].

\section{Waldenström's Macroglobulinemia—a scylla like heterogenous lymphoma}

\section{Clinical characteristics}

76 years have passed since Jan Waldenström reported about a disease, named later Waldenström's Macroglobulinemia that was characterized besides salient clinical features like fatigue, epistaxis, and lymphadenopathy by bone marrow lymphocytosis and an increased amount of serum macroglobulin [37]. Already a few years earlier Bing and Neel had reported about three cases with macroglobulinemia and central nervous affection, these cases are designated as Bing-Neel syndrome [38, 39]. Since then tremendous progress has been made in characterizing the underlying biology and genetic landscape in WM, but still today WM is defined as clinicopathological entity characterized by the presence of a lymphoplasmacytic lymphoma in the bone marrow (BM) and an elevated monoclonal serum IgM level $[40,41]$. The cellular composition of WM comprises malignant lymphocytes and plasma cells, forming a picture of a scylla-like heterogenous hybrid disease. Its clinical picture is accordingly heterogenous characterized by constitutional symptoms, anemia, fatigue as well as IgM related symptoms such as hyperviscosity or neuropathy [42, 43]. WM is rare, with an incidence rate of 4-7 per 1 million European women and men per year, respectively [44], presenting $1-2 \%$ of all hematological disorders. It is a disease of the elderly and occurs more frequently in male than in female and in Caucasians than in African Americans [45]. WM is incurable and its cause is unknown, however, evidence for a high familial incidence is compelling and approximately one-fourth of patients have family members with a history of lymphoproliferative neoplasias [46]. WM is often originating from IgM-monoclonal gammopathy of undetermined significance (MGUS) [47] and transformation into aggressive diffuse large B-cell lymphoma (DLBCL) [48] as well as leukemia [49] has been reported. WM has an indolent clinical cause in the majority of cases and mortality varies in patients with WM. Overall survival rates (OS) at 10 years are around $40 \%$ with a median age of 69 at diagnosis. In another analysis the relative survival (taking the expected survival of the general population in a specific calendar year into account) was $57 \%$, based on the Surveillance, Epidemiology, and End Results (SEER) database and including patients with WM diagnosed between 1980 and $2010(n=7744)[50,51]$. However longer survival rates have been observed in patients $<45$ with 10 -year OS rates of $86 \%$ [52]. Main causes of death being progression, transformation to a high-grade lymphoma, or therapyrelated complications [50].

\section{Genetic landscape}

Since Burnet who postulated genetic factors in the form of mutations might be basis of neoplasms with abnormal paraprotein production, many genetic abnormalities have been linked to WM [53]. In the 70ties Spencer et al. remarkably illustrated his hypothesis in a case study with twins of which one twin acquired WM whereas the other was healthy, the one affected individual showing an acquisition of an extra Chromosome A, linking this chromosomal gain to WM [54]. A few years later this finding was confirmed and found in 3 additional patients [55], therefore emphasizing that a hereditary pre-disposition might be an important contributing factor in this disease. Today's evidence for a familial predisposition in WM is 
striking mapping $26 \%$ of WM patients to family members with either WM or another B-cell disorder in a large singlecenter study evaluating 924 cases [56].

Beside an obvious familial disposition first array-based genomic hybridization analysis demonstrated that $83 \%$ of the WM patients carry chromosomal abnormalities. Chromosome $6 q$ 21-25 deletions are the most frequent observed aberrations, affecting $30 \%$ of patients [57], whereas gains in $6 \mathrm{p}$ are observed in every $5^{\text {th }}$ patient and are mainly described as a subsequent event of $6 q$ deletions [58]. Further cytogenetic disorders include $13 \mathrm{q} 14$ and $17 \mathrm{p}$ deletions as well as trisomy 4, 12, and 18 [59]. Research also has directed attention towards the role of microRNAs in the biology of WM as well as to the impact of epigenetic alterations such as histone acetylation downregulating microRNAs such as miRNA-9* [60]. An increased expression has been identified for miRNAs-363*, $-206,-494,-155,-184$, and $-542-3 p$ of which mi-RNA-155 was further validated and proved as growth-promoting oncogenic factor in the WM cell line BCWM.1 [61].

\section{The CXCR4 mutation-a major driver in WM biology}

The most recent and striking development in WM genetics lead to the identification of highly recurrent somatic mutations in two genes, namely MYD88 and CXCR4, which clearly has paved the way to a deepened understanding of the signaling cascades driving growth and importantly also therapeutic resistance in WM [62].

MYD88 mutations occur mainly monoallelic in over $90 \%$ of patients with WM and with this are by far most common though not specific for this lymphoma subtype [63]. They also occur at very low frequencies in diffuse large B-cell lymphoma (DLBCL), marginal zone lymphoma (MZL), and chronic lymphocytic leukemia (CLL), but allow differentiation to other lymphomas such as IgM multiple myeloma (MM), which virtually never shows MYD88 mutations [64]. The vast majority of mutations change the $\mathrm{T}$ by $\mathrm{C}$ at position 265 (MYD88 point mutation L265P), but alternate mutations occur as subclonal event in $C$ to $G$ resulting in an amino acid substitution (S219C) (1 patient) or alternative mutations M232T and S243N [58, 59]. A so far unknown mutation was recently described to cause the substitution of L265 with RPP (L265RPP). MYD88 is a protein adapter that contains three main domains including a death domain (DD), an intermediate linker domain (ID), and the toll/interleukin-1 receptor domain (TIR) at the $\mathrm{C}$ terminus. Upon recruitment from Toll-like receptor (TLR), MYD88 activates the IL-1 receptor signaling pathway via interleukin-1 receptor-associated kinases 1,2 , and 4 (IRAK1, IRAK2, IRAK4) leading to a phosphorylation and constitutive activation of BTK (Bruton's tyrosine kinase). $\mathrm{BTK}$, a critical kinase in B-cell receptor (BCR) signaling that regulates immune response, cell proliferation, and cell death through activation of the NF-KB signaling pathway (p50-p65) and the mitogen-activated protein kinase (MAPK) pathway. BTK interacts with four different proteins downstream of TLR signaling including TIR, MYD88, IRAK1, and TIRAP/MAL (TIR domain containing adapter protein TIRAP or MyD88 adapter-like (MAL)). In WM the level of activated BTK is higher when MYD88 is mutated compared to the unmutated cells. The biology of MYD88 mutations has been reviewed in depth elsewhere [65-67].

The second most recurrent mutation in WM hits the CXCR4 gene: mutations in this gene can be found in up to $40 \%$ of the patients. In total more than 40 mutations were described so far [68], all exclusively found in the regulatory cytosolic domain stretching from amino acid position 308 to 352. Unlike MYD88, multiple CXCR4 mutations can be present in an individual appearing with heterozygous character in different clones [59].

The most frequent $(50 \%)$ mutated region is the amino acid S338X at position 1013 with nucleotide changes $\mathrm{C}>\mathrm{G}$ in $54 \%$ and $\mathrm{C}>\mathrm{A}$ in $25 \%$ of the cases, both resulting in a stop codon, followed by S338 frameshift mutation in $21 \%$ of the cases. Almost all mutations described in WM introduce a premature stop codon or a frameshift that impair CXCR4 desensitization and internalization, thereby prolonging signaling upon binding of the chemokine ligand CXCL12 (Fig. 2) [58, 69].

The main signaling axis that is affected by CXCR4 mutations promote enhanced AKT and subsequent MAPK $1 / 2$ signaling, resulting in sustained survival signals for cancer cells [70]. Perpetual signaling enhanced by nonsense as well as frameshift mutations have been investigated with regard to their function in WM. Stimulation of engineered WM cells with CXCL12 resulted in significantly reduced

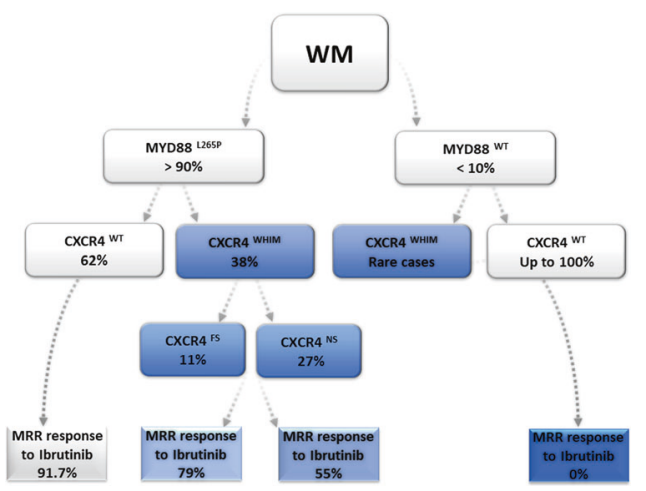

Fig. 2 MYD88 and CXCR4 mutations in WM. Summary of the distribution of MYD88 and CXCR4 mutations in WM (according to $[58,68,76,77])$. MRR are given for Ibrutinib (for MYD88 $8^{\mathrm{L} 265 \mathrm{P}}$, $\mathrm{CXCR}^{\mathrm{WT}}$, MYD88 ${ }^{\mathrm{WT}} / \mathrm{CXCR} 4^{\mathrm{WT}}$ according to $[76,77](n=62)$ and for CXCR4 FS $(n=49)$ versus NS $(n=19)$ according to [68]) NS nonsense, FS frameshift, WT wildtype WHIM Warts, Hypogammaglobulinemia, recurrent bacterial Infections and Myelokathexis, MRR major responds rate. 
levels of CXCR4 receptor internalization compared to the wildtype control. Both frameshift and nonsense mutation potentiate MAPK signaling following CXCL12 stimulation when compared to the wildtype. Direct comparison of frameshift versus nonsense CXCR4 mutations reveal an enhanced MAPK signaling for the nonsense mutation S338X. The results confirm clinical observations of CXCR4 S338X showing prolonged MAPK signaling compared to frameshift mutations and present with higher tumor burden. The reason for the variation of MAPK1 activation is still in abeyance but possible explanations might be the loss of CXCR4 C-terminal binding sites for $\beta$-arrestin that sustains MAPK1 signaling or a difference in CXCR4 dimer formation and subsequent downstream signaling. When testing sensitivity to ibrutinib, CXCR4 mutant-expressing cells were rescued by CXCL12 from ibrutinib-induced apoptosis and the effect could be abrogated by adding plerixafor [71]. By determining the cancer cell fraction (CCF) of CXCR4 mutations in WM the mutation S338X was identified as mostly sub-clonal with variable clonal distribution, which points to the mutation as a late oncogenic event acquired after MYD88 mutation. The analysis was performed using parallel quantitative AS-PCR analyses for MYD88 L265P and CXCR4 S338X from 21 untreated WM patients resulting in a highly clonal distribution. With 13 patients the majority expressed CXCR4 S338X C > G only and when analyzed in relation to MYD88 L265P showed a clonal distribution of $44.5 \%$. For $7 \mathrm{WM}$ patients who expressed both CXCR4 S338X mutations $(\mathrm{C}>\mathrm{G}$ and $\mathrm{C}>\mathrm{A})$, the fraction of cells expressing CXCR4 S338X relative to MYD88 L265P was around 4\% [72, 73]. In contrast to data on CXCR4 mutations data on the expression levels of CXCR4 are scarce in WM compared to normal counterparts. However, it could be demonstrated that transcription of CXCR4 is high in WM particularly in patients with $\mathrm{MYD} 8^{\mathrm{MUT}} / \mathrm{CXCR} 4^{\mathrm{WT}}$ compared to circulating normal B cells [74].

\section{Impact of CXCR4 mutations on treatment outcome in WM patients}

Patients with CXCR4 mutations are confronted with the downside of showing higher bone marrow involvement, higher IgM levels, symptomatic hyperviscosity, and a more aggressive disease at diagnosis with reduced sensitivity toward the BTK-inhibitor ibrutinib. Of note, CXCR4 mutations occur already in asymptomatic WM and the presence of these mutations was associated with a shorter treatment-free survival compared to patients without CXCR4 mutation [75].

Data particularly from trials testing the BTK inhibitor ibrutinib have shown that CXCR4 mutations can impact clinical outcome in patients with WM, underlining the key importance of this gene also in the clinical setting. The following sections describe our current knowledge on the influence of the CXCR4 mutational status on therapy outcome in this disease (summarized in Table 1).

\section{BTK inhibitors}

Targeting the BTK in WM cells by BTK inhibitors has changed the treatment landscape in WM: in the pivotal phase II study 63 symptomatic relapsed patients with Waldenström's macroglobulinemia received Ibrutinib at a daily oral dose of $420 \mathrm{mg}$ until disease progression or the development of unacceptable toxicity. This oral singleagent treatment resulted into an overall response rate of $90.5 \%$ and a major response rate of $73 \%$, characterizing ibrutinib as the most efficient single-agent treatment in WM. Of note, responses varied with regard to the mutational status of MYD88 and CXCR4 with highest responses in the MYD88 ${ }^{\mathrm{Mut}} / \mathrm{CXCR} 4^{\mathrm{WT}}$, intermediate responses in the MYD88 $8^{\text {Mut }} / \mathrm{CXCR} 4^{\text {Mut }}$ and lowest responses in the MYD88 ${ }^{\mathrm{WT}} / \mathrm{CXCR} 4^{\mathrm{WT}}$ cases (Fig. 2). In particular nonsense CXCR4 mutations in contrast to frameshift mutations were associated with delayed time to response, less deep responses, and shorter PFS upon treatment with ibrutinib monotherapy [68]. The estimated 2-year progression-free and overall survival rates among all patients were $69.1 \%$ and $95.2 \%$, respectively. Treatment-related toxic effects of grade 2 or higher were similar as observed in other related lymphomas such as chronic lymphocytic leukemia or mantle cell lymphoma and included neutropenia (in $22 \%$ of the patients) and thrombocytopenia (in 14\%). Postprocedural bleeding and atrial fibrillation associated with a history of arrhythmia occurred in 3\% and 5\% of cases, respectively [69, 76, 77]. A prospective study for untreated WM patients, in which all 30 patients were MYD88 mutated and $47 \%$ had additional CXCR4 mutations, confirmed the results. Overall and major responses for all patients were $100 \%$ and $83 \%$, respectively. As seen in relapsed patients responses were depending on the mutational status with a drop in major $(94 \% \mathrm{v} 71 \%)$ and very good partial $(31 \mathrm{v} 7 \%)$ responses for patients with mutated CXCR4 and delayed time to major responses in this patient group (1.8 v 7.3 months; $P=0.01$ ) [64]. Responses to Ibrutinib and also PFS depends also on the allelic burden of CXCR4 as was shown in a smaller retrospective analysis of 37 patients treated with ibrutinib: in patients with CXCR $4^{\text {S338X }}$ mutations, clonality of $\geq 25 \%$ was associated with lower response rates and inferior PFS compared to patients with $<25 \%$ clonality [72]. Based on the observation, that ibrutinib single agent has less activities in CXCR4 mutated patients and in patients with nonmutated MYD88 and CXCR4, a large international prospective study performed on behalf of the European 


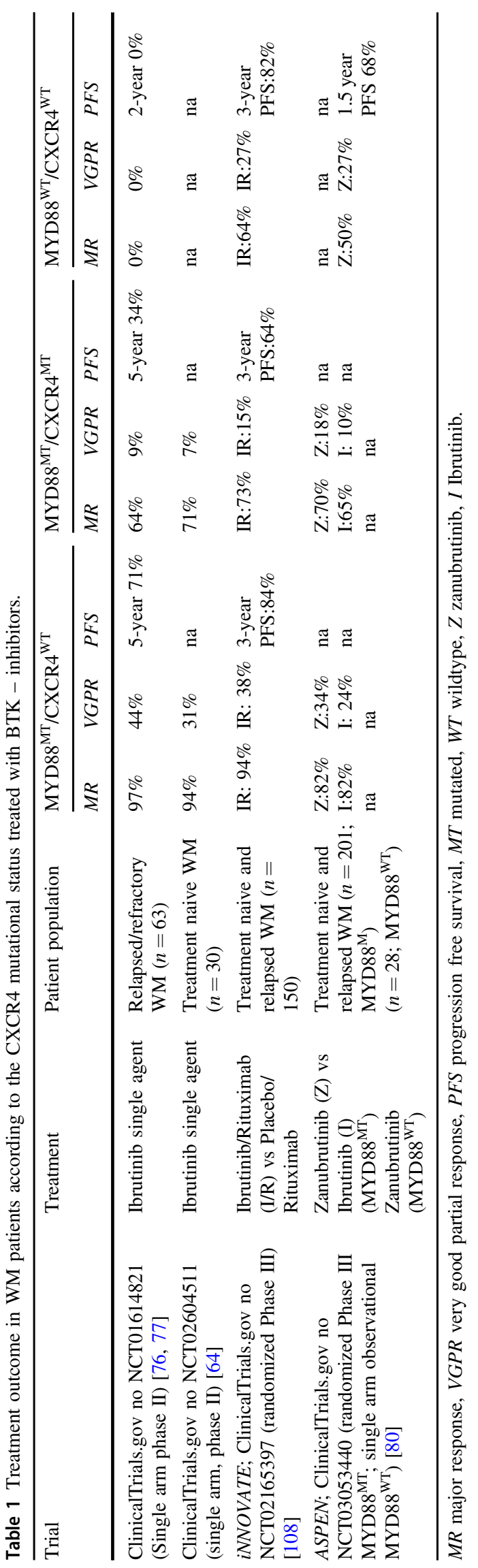

Consortium for Waldenström's Macroglobulinemia (ECWM) was initiated, randomizing 150 patients with treatment naive or pretreated WM between ibrutinib plus rituximab or placebo plus rituximab. Primary endpoint was PFS, which was significantly superior in the ibrutinib arm at 30 months (82\% with ibrutinib-rituximab versus $28 \%$ with placebo-rituximab; hazard ratio for progression or death, $0.20 ; P<0.001$ ) (Fig. 3). Importantly, addition of rituximab to ibrutinib resulted into a largely homogenous response rate and PFS independent of the mutational status of MYD88 and CXCR4, demonstrating that the combination can increase response, time to response, and PFS in WM with double mutated MYD88 and CXCR4 as well as in WM with both genes non-mutated (Fig. 2). Atrial fibrillation and hypertension of grade 3 or higher occurred more frequently with ibrutinib-rituximab than with placebo-rituximab ( $12 \%$ vs. $1 \%$ and $13 \%$ vs. $4 \%$, respectively); in contrast, infusion reactions and any grade of IgM flare occurred less frequently in the ibrutinib arm (1\% vs. $16 \%$ and $8 \%$ vs. $47 \%$, respectively). Based on these data ibrutinib in combination with rituximab was approved for treatment-naive and relapsed patients with WM by the FDA and the EMA [78].

Second generation BTK inhibitors are characterized by less off target effects and aim at increasing efficacy and reducing side effects seen with ibrutinib. More recently, a randomized Phase II trial reported excellent results in 102 patients with treatment naive or relapsed WM with singleagent acalabrutinib, given $100 \mathrm{mg}$ twice a day until progression or non-tolerated toxicity. After a median follow-up of 27.4 months response rate was $93 \%$ for treatment naive patients and $93 \%$ for relapsed/refractory patients. There seemed to be less atrial fibrillation compared historically to ibrutinib. Data on the impact of CXCR4 mutations were preliminary in this study as there was no central laboratory assessment of CXCR4 mutations in this study [79]. Zanubrutinib, another second generation BTK inhibitor has shown encouraging data in CLL and in a limited number of WM patients and was recently tested in a randomized phase III trial in MYD88 mutated patients in a head-to-head comparison with single-agent ibrutinib. Responses were deeper with Zanubrutinib, but did not translate into a longer PFS or OS in this study, although the follow-up at the time of presentation was still short with 19.4 months. A post-hoc analysis of baseline bone marrow from 190 (95\%) patients using next generation sequencing (NGS) for CXCR4 mutation detection revealed the presence of $\mathrm{CXCR} 4{ }^{\mathrm{WHIM}}$ mutations in $53(28 \%)$ of all patients. VGPR rates demonstrated a higher rate overall of $29 \%$ versus $18 \%$ for $\mathrm{CXCR} 4{ }^{\mathrm{WT}}$ versus CXCR $4{ }^{\mathrm{WHIM}}$ patients, a trend also seen in the ibrutinib arm [80]. Longer follow-up of the aforementioned study is needed to confirm whether these new BTK inhibitors are indeed more efficient and/or less toxic 
Fig. 3 Progression-free survival of WM patients treated with ibrutinib/ rituximab versus placebo/ rituximab. The iNNOVATE phase III randomized study (a) for the total patient group and (b) depending on the MYD88/CXCR4 mutational status [108].

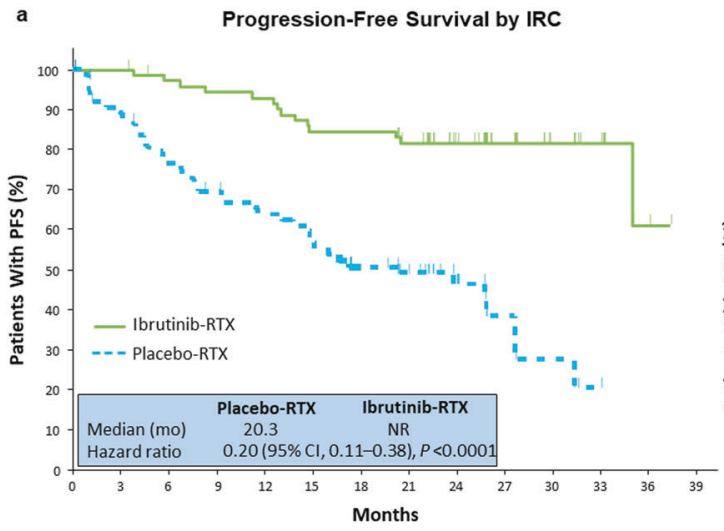

b Progression-Free Survival by Genotype

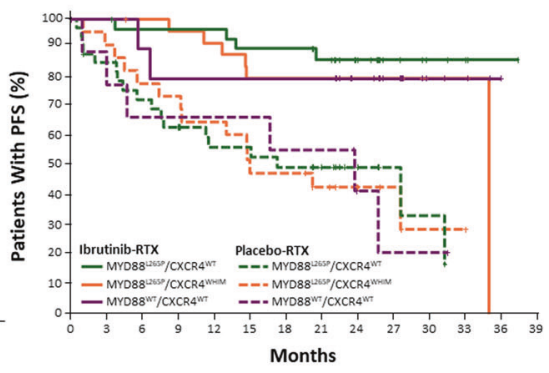

compared to ibrutinib in WM. Taken together, also in CXCR4 mutated patients BTKi stays an important backbone of treatment, but addition of Rituximab to ibrutinib or a second-generation BTKi should be considered in these patients, in particular in the case of delayed and/or insufficient response.

\section{Proteasome Inhibitors}

Among the proteasome inhibitors bortezomib is widely used for the treatment of WM based on several phase II trials demonstrating efficacy of bortezomib used as a single agent or in combination with rituximab in WM [81-83]. Intriguingly, so far retrospective data suggest that Bortezomib acts independent of the MYD88 and CXCR4 mutational status: in a retrospective analysis of a phase II study comprising 63 treatment naive or relapsed patients treated with bortezomib/rituximab (bortezomib IV weekly at 1.6 $\mathrm{mg} / \mathrm{m}^{2}$ on days 1,8 , and 15 , every 28 days 36 cycles, and rituximab $375 \mathrm{mg} / \mathrm{m}^{2}$ at days $1,8,15$, and 22 , on cycles 1 ) PFS and OS was independent of the CXCR4 mutational status in MYD88 mutated patients. In this retrospective analysis 43 patients were evaluable for CXCR4 mutations with 17 patients being CXCR4 mutated. All CXCR4 mutated patients carried also the MYD88 L265P mutation. Thus, this study did not allow to test the efficacy in MYD88 non-mutated WM [84]. Recent data of a phase I/II study of the ECWM using the orally applicable proteasome inhibitor ixazomib in combination with dexamethasone and rituximab also induced comparable response rates and PFS in CXCR4 mutated versus wildtype cases, although the patient number was limited with 59 patients evaluable in total and $88 \%$ of patients with a MYD88L265P mutation and $35 \%$ with a CXCR4 mutation [85]. In a recently published study, 26 treatment naïve WM patients received a combination of ixazomib, dexamethasone and rituximab (IDR) for 6 monthly induction cycles followed by 6 every-2-month maintenance cycles. All the patients carried a MYD 88 $L 265 P$ mutation and from these $58 \%$ a $C X C R 4$ mutation.
Positivity for the CXCR4 mutation delayed the median time to response (TTR) and time to major response (TTMR) (median TTR of 3 and 1 month, respectively $(p=0.003)$, and median TTMR of 10 and 3 months, respectively $(p=$ 0.31)). Also the rate of VGPR were lower in CXCR4 mutated patients (with and without CXCR4 mutations 7\% and $36 \%$, respectively $(p=0.06))$, whereas the median PFS, duration of response and time to next treatment was not affected [86]. Another proteasome inhibitor sparing neurotoxicity associated with bortezomib is carfilzomib. Carfilzomib was evaluated in combination with rituximab and dexamethasone (CaRD) in WM patients that were naive to both Bortezomib and rituximab [87]. CaRD therapy consisted of IV Carfilzomib given at $20 \mathrm{mg} / \mathrm{m}^{2}$ (on cycle 1), and on $36 \mathrm{mg} / \mathrm{m}^{2}$ thereafter (cycles 2-6), on days 1, 2 and 8 , 9 with dexamethasone $20 \mathrm{mg}$ on days $1,2,8,9$ and rituximab $375 \mathrm{mg} / \mathrm{m}^{2}$ on days 2, 9 every 21 days. Maintenance therapy followed 8 weeks after cycle 6 with IV Carfilzomib $36 \mathrm{mg} / \mathrm{m}^{2}$ and dexamethasone $20 \mathrm{mg}$ on days 1,2 and rituximab $375 \mathrm{mg} / \mathrm{m}^{2}$ on day 2 every 8 weeks for 8 cycles. The overall response rate in this study was $87 \%$, and $68 \%$ of the patients achieved a major response. In this study, MYD88 and CXCR4 tumor mutational status were examined and did not appear to impact response attainment, although the patient numbers are small. The impact of carfilzomib in combination with ibrutinib will be tested in a large international phase III study of the ECWM, randomizing treatment naive or relapsed patients molecularly profiled for MYD88 and CXCR4 mutations between carfilzomib/ibrutinib versus ibrutinib (Clinicaltrials.gov No. NCT04263480).

\section{Rituximab/chemotherapy}

Data on the impact of CXCR4 mutations on the outcome of rituximab/chemotherapy are scarce: in a multi-center retrospective study performed between January 2013 and December 2017 in France 69 patients were enrolled. 45 of $51(88 \%)$ and 11 of 44 tested patients $(25 \%)$ were mutated 
Table 2 CXCR4 antagonists and antibodies under clinical investigation (source ClinicalTrials.gov).

\begin{tabular}{|c|c|c|c|c|}
\hline Drug & Trial phase & Status & Condition & $\begin{array}{l}\text { ClinicalTrials.gov } \\
\text { identifier }\end{array}$ \\
\hline BKT140 & Phase I/II & Completed & Multiple Myeloma & NCT01010880 \\
\hline $\begin{array}{l}\text { BMS-936564 } \\
\text { Lenalidomide } \\
\text { Dexamethasone } \\
\text { Bortezomib }\end{array}$ & Phase I & Completed & Multiple Myeloma & NCT01359657 \\
\hline $\begin{array}{l}\text { Ulocuplumab } \\
\text { Lenalidomide } \\
\text { Dexamethasone } \\
\text { Bortezomib }\end{array}$ & Phase Ib/II & Completed & Multiple Myeloma & NCT01359657 \\
\hline $\begin{array}{l}\text { Ulocuplumab } \\
\text { Ibrutinib }\end{array}$ & Phase I/II & Active & WM & NCT03225716 \\
\hline $\begin{array}{l}\text { Mavorixafor } \\
\text { Ibrutinib }\end{array}$ & Phase I & Recruiting & WM & NCT04274738 \\
\hline BMS-936564 & Phase I & Completed & AML, DLBCL, CLL FL & NCT01120457 \\
\hline Plerixafor & & Recruiting & MM & NCT03406091 \\
\hline $\begin{array}{l}\text { BL-8040 } \\
\text { (BKT-140) } \\
\text { Nelarabine }\end{array}$ & Phase II & Recruiting & T-ALL & NCT02763384 \\
\hline TG-0054 & Phase I & Completed & Healthy & NCT00822341 \\
\hline Plerixafor & Phase II & Completed & Healthy & NCT00075335 \\
\hline Plerixafor & $\begin{array}{l}\text { Prospective } \\
\text { observational }\end{array}$ & Completed & $\begin{array}{l}\text { Lymphoma } \\
\text { Myeloma }\end{array}$ & NCT01700608 \\
\hline $\begin{array}{l}\text { Plerixafor } \\
\text { G-CSF }\end{array}$ & Phase II/III & $\begin{array}{l}\text { Active, not } \\
\text { recruiting }\end{array}$ & WHIM & NCT02231879 \\
\hline Plerixafor & Phase I/II & Recruiting & WHIM & NCT00967785 \\
\hline $\begin{array}{l}\text { Plerixafor } \\
\text { Mitoxantrone } \\
\text { Etoposide } \\
\text { Cytarabin }\end{array}$ & Phase I/II & Completed & AML & NCT00512252 \\
\hline Plerixafor & Phase I/II & Completed & AML & NCT01141543 \\
\hline POL6326 & Phase I & Completed & Healthy & NCT01841476 \\
\hline $\begin{array}{l}\text { BL-8040 } \\
\text { Ara-C }\end{array}$ & Phase II & Completed & AML & NCT01838395 \\
\hline
\end{tabular}

for MYD88 and CXCR4, respectively. The regimen induced an overall (ORR) of 97\% and a major (MRR) response of $96 \%$. Thirteen (19\%) patients achieved a complete response (CR), 26 (37\%) very good partial response (VGPR), $28(40 \%)$ partial response (PR) and 1 (1\%) MR; one patient had stable disease. Of note, disease response was not different between patient with or without CXCR4 mutation [88].

\section{Targeting CXCR4 as an innovative therapeutic approach}

Based on the role of CXCR4 as an oncogenic factor in a variety of cancers, targeting of CXCR4 has been recognized as a highly attractive therapeutic principle since many years. Initially, CXCR4 antagonists were identified and developed for HIV treatment as CXCR4 acts as a co-factor for cell entry of the virus. CXCR4 antagonists can be separated into four groups: peptides, such as EPI-X-4 or T140, nonpeptides such as the clinical approved biclam AMD3100, antibodies against CXCR4 and optimized agonists and antagonist [89].

Peptides and peptide derivatives have been used first to develop CXCR4 antagonists. Initially, the group of polyphemusin peptides was discovered screening naturally occurring peptides from horseshoe crabs for HIV entry blocking capacity [89]. Chemical modification has led to BKT140 also known as BL-8040 (TN14003) which is an analog of $\mathrm{T} 140$ and in focus of recent pre-clinical and clinical trials in patients with leukemia and lymphoma [89] (Table 2). Recent research has focused on exploring peptide libraries to identify promising bioactive peptides from human sources [90, 91]. Screening of the hemofiltrate had been successful in 2015 when Zirafi and colleagues identified a, so far unknown highly specific CXCR4 antagonist named EPI-X4. The 16-mer peptide EPI-X4 is a segment of 
albumin and results from resecting Cathepsins under acidic conditions which is a characteristic of inflammation. Cathepsins are secreted from nearly all cell types and it is known that Cathepsin D expression is high in B cell lymphomas [92]. In contrast to AMD3100 EPI-X4 does not bind CXCR7 and has no mitochondrial cytotoxicity, suggesting lower side effects. Intense research has already shown that EPI-X4 is able to interfere in the crosstalk between CXCR4 and CXCL12, inhibits HIV-1 infection, mobilizes hematopoietic stem cells and is able to suppress migration of leukemic cells in vitro [12] (Fig. 4).

Bicyclams belong to the non-peptidic group of small molecules that target CXCR4. Within this group AMD3100 represents the most prominent and known molecule which got approved by the FDA in 2008 for mobilization of hematopoietic stem cells of non-Hodgkin lymphoma and multiple myeloma [93]. Another approach to target CXCR4 is the development of antibodies directed against CXCR4. Here, BMS-936564 / MDX1338 also called Ulocuplumab belongs to the most promising candidates and is currently under investigation in clinical phase I, including a phase $\mathrm{Ib} /$ II trial in multiple myeloma, combining the anti-CXCR4 antibody with lenalidomide or bortezomib plus dexamethasone, showing encouraging clinical activity [94, 95]. A selection of CXR4 antagonists and antibodies and their stage of development in terms of hematologic neoplasms can be found in Table 2 .

In WM the appealing approach to counteract constitutive activation mutated CXCR4 by CXCR4 antagonists has just begun to be tested in clinical trials. Results from a

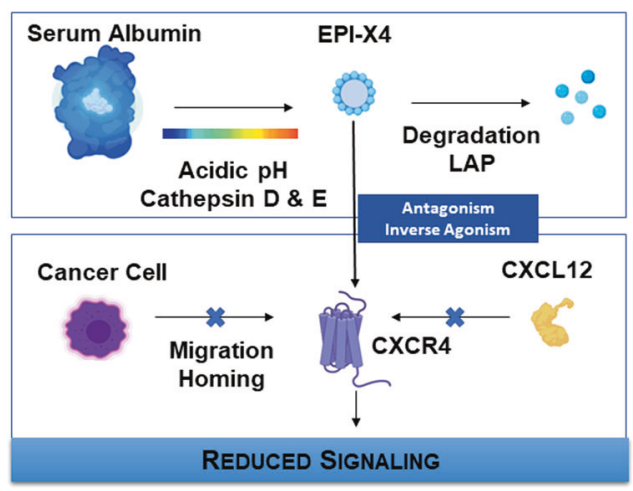

Fig. 4 Summary of the generation, interaction, and function of the CXCR4 antagonist EPI-X4. The endogenous peptide EPI-X4 is cleaved from highly abundant serum albumin under low $\mathrm{pH}$ conditions. The generation is controlled by aspartatic proteases Cathepsin D and $\mathrm{E}$ and stopped by leucylaminpeptidases (LAPs) within a relatively short time-frame. After its release EPI-X4 binds to CXCR4 in a very specific manner and competes with CXCL12 binding, acting as an antagonist. Its inverse agonist ability furthermore suppresses constitutive signaling activity of CXCR4 in the absence of CXCL12. By cordoning off the interaction between CXCR4 and its agonist CXCL12, EPI-X4 mobilizes hematopoietic stem cells and is able to suppress migration and homing of cancer cells. prospective phase I/II study evaluating ibrutinib in combination with the anti-CXCR4 monoclonal antibody ulocuplumab in WM patients who carry CXCR4 mutations, are awaited soon (NCT03225716).

Ulocuplumab inhibits the binding of CXCR4 to CXCL12, and has shown both in vitro as well as in vivo anti-tumor in a WM tumor xenograft model engrafted with CXCR $4{ }^{\text {WHIM }}$ mutated tumor cells [96]. Ulocuplumab demonstrates pharmacokinetics which allows weekly induction, and bi-weekly maintenance therapy, which stands in contrast to the short half-life of plerixafor [97]. Based on these data a prospective Phase I/II clinical study of ulocuplumab with ibrutinib was started for WM patients with CXCR4 mutation using three different dose cohorts with the goal to improve ibrutinib responses in CXCR4 mutated patients. The same concept follows a just recently activated phase $1 \mathrm{~b}$ multi-center, open-label, dose-escalation trial assessing the safety and tolerability of mavorixafor in combination with ibrutinib in MYD88 mutated WM patients harboring an additional CXCR4 mutation (NCT04274738). Changes in serum immunoglobulin $M$ (IgM) and hemoglobin ( $\mathrm{Hgb})$ from baseline will be used as biomarkers of clinical response. The clinical trial is expected to enroll $\sim 12-18$ patients and is conducted as part of a collaboration with The Leukemia \& Lymphoma Society to accelerate the development of mavorixafor for the treatment of WM. Mavorixafor (AMD070) is a potent, selective, and orally bioavailable CXCR4 chemokine receptor allosteric antagonist with an IC50 value of $13 \mathrm{nM}$ against CXCR4 125I-SDF binding [98].

\section{Future developments}

WM is an example, which demonstrates that insights into the biology has been successfully translated into clinical concepts as shown for the class of BTK inhibitors as well as for CXCR4 inhibitors. As we can state already that BTK inhibitors have changed the treatment landscape in WM, it is too early to say this for the CXCR4 inhibitors. But first data are indeed encouraging and a success of this class of compounds in WM would be another excellent proof that understanding the biology of WM is the key which opens the doors to innovative treatments. CXCR4 inhibitors will be not the end of the road. There were early data on BCL-2 expression in WM independently of the genotype and first data have shown promising activity of the BCL-2 inhibitor venetoclax in relapsed/refractory MYD88 and CXCR4 mutated patients with WM [99, 100]. In addition, WM comprises a cellular compartment with plasmacytic differentiation, being responsible for the IgM production of the malignant clone. These cells are positive for $\mathrm{CD} 38$ and trials are ongoing testing daratumumab in patients with WM. Another fascinating and greatly promising novel approach is the CAR-T 
cell therapy, which has shown highly promising activity in lymphoma subtypes such as DLBC and MCL [101-103]. Data in indolent lymphoma are still limited with first encouraging data reported for follicular lymphoma [104]. In a case report a patient with transformed WM was successfully treated with CAR-T cells, notably also with eradication of the indolent lymphoma residues [105]. Beside these implications for treatment the MYD88 and CXCR4 mutational status can also guide diagnostics: thus, it is known that MYD88 mutations can help to discriminate WM from IgM positive $\mathrm{MM}$, as the latter one is virtually in all cases MYD88 ${ }^{\mathrm{WT}}$ [106]. In addition, presence of the CXCR4 mutation can be indicative for WM, but is not specific and was described in in rare cases of DLBCL prolymphocytic leukemia and follicular lymphoma [107] (https://dcc.icgc.org/). All this exemplifies that the future in WM looks bright based on a dynamic research community which drives translational research and by this paves the way to a better clinical management of WM patients.

Acknowledgements The work was supported by the grant from the DFG (German Research Foundation) SFB 1279 project B01 to L.M.K. and C.B. Open Access funding enabled and organized by Projekt DEAL.

\section{Compliance with ethical standards}

Conflict of interest The authors declare the following competing interests: L.M.K. none; C.B. received honoraria from Roche, Pharmacyclics, Janssen, Beigene, AbbVie and received research funding from Roche, Janssen, MSD, Bayer. Z.M.H. holds an institutional patent issued for detection of CXCR4 mutations for diagnostic and treatment of WM; S.P.T. received research support from Pharmacyclics/Abbvie, Bristol Myers Squibb, X4 Pharmaceuticals, Beigene and Eli Lilly as well as consultation fees from Pharmacyclics/Abbvie, Janssen, and Beigene.

Publisher's note Springer Nature remains neutral with regard to jurisdictional claims in published maps and institutional affiliations.

Open Access This article is licensed under a Creative Commons Attribution 4.0 International License, which permits use, sharing, adaptation, distribution and reproduction in any medium or format, as long as you give appropriate credit to the original author(s) and the source, provide a link to the Creative Commons license, and indicate if changes were made. The images or other third party material in this article are included in the article's Creative Commons license, unless indicated otherwise in a credit line to the material. If material is not included in the article's Creative Commons license and your intended use is not permitted by statutory regulation or exceeds the permitted use, you will need to obtain permission directly from the copyright holder. To view a copy of this license, visit http://creativecommons. org/licenses/by/4.0/.

\section{References}

1. Caruz A, Samsom M, Alonso JM, Alcami J, Baleux F, Virelizier $\mathrm{JL}$, et al. Genomic organization and promoter characterization of human CXCR4 gene. FEBS Lett. 1998;426:271-8.
2. Hughes CE, Nibbs RJB. A guide to chemokines and their receptors. FEBS J. 2018;285:2944-71.

3. Wu B, Chien EYT, Mol CD, Fenalti G, Liu W, Katritch V, et al. Structures of the CXCR4 chemokine receptor in complex with small molecule and cyclic peptide antagonists. Sci (NY). 2010; 330:1066-71.

4. Pawig L, Klasen C, Weber C, Bernhagen J, Noels H. Diversity and inter-connections in the CXCR4 chemokine receptor/ligand family: molecular perspectives. Front Immunol. 2015;6:429.

5. Shirozu M, Nakano T, Inazawa J, Tashiro K, Tada H, Shinohara T, et al. Structure and chromosomal localization of the human stromal cell-derived factor 1 (SDF1) gene. Genomics. 1995;28:495-500.

6. Greenbaum A, Hsu YM, Day RB, Schuettpelz LG, Christopher MJ, Borgerding JN, et al. CXCL12 in early mesenchymal progenitors is required for haematopoietic stem-cell maintenance. Nature. 2013;495:227-30.

7. Nagasawa T, Hirota S, Tachibana K, Takakura N, Nishikawa S, Kitamura Y, et al. Defects of B-cell lymphopoiesis and bonemarrow myelopoiesis in mice lacking the CXC chemokine PBSF/SDF-1. Nature. 1996;382:635-8.

8. Teicher BA, Fricker SP. CXCL12 (SDF-1)/CXCR4 pathway in cancer. Clin Cancer Res. 2010;16:2927-31.

9. Saini V, Marchese A, Majetschak M. CXC chemokine receptor 4 is a cell surface receptor for extracellular ubiquitin. J Biol Chem. 2010;285:15566-76.

10. Qin L, Kufareva I, Holden LG, Wang C, Zheng Y, Zhao C, et al. Structural biology. Crystal structure of the chemokine receptor CXCR4 in complex with a viral chemokine. Science. 2015;347: 1117-22.

11. De Clercq E. Inhibition of HIV infection by bicyclams, highly potent and specific CXCR4 antagonists. Mol Pharmacol. 2000; 57:833-9.

12. Zirafi O, Kim KA, Standker L, Mohr KB, Sauter D, Heigele A, et al. Discovery and characterization of an endogenous CXCR4 antagonist. Cell Rep. 2015;11:737-47.

13. Bao S, Li T, Long X, Zhang J, Zhao H, Ren Y, et al. Chemokine receptor type 4 regulates migration and invasion of trophectoderm cell in the human blastocyst. Biol Reprod. 2016;95:21.

14. Tachibana K, Hirota S, Iizasa H, Yoshida H, Kawabata K, Kataoka $\mathrm{Y}$, et al. The chemokine receptor CXCR4 is essential for vascularization of the gastrointestinal tract. Nature. 1998;393:591-4.

15. Miller RJ, Banisadr G, Bhattacharyya BJ. CXCR4 signaling in the regulation of stem cell migration and development. J Neuroimmunol. 2008;198:31-8.

16. Rossi D, Zlotnik A. The biology of chemokines and their receptors. Annu Rev Immunol. 2000;18:217-42.

17. Singh P, Mohammad KS, Pelus LM. CXCR4 expression in the bone marrow microenvironment is required for hematopoietic stem and progenitor cell maintenance and early hematopoietic regeneration after myeloablation. Stem Cells. 2020;38:849-59.

18. Balkwill F. The significance of cancer cell expression of the chemokine receptor CXCR4. Semin Cancer Biol. 2004;14:171-9.

19. Chatterjee S, Behnam Azad B, Nimmagadda S. The intricate role of CXCR4 in cancer. Adv Cancer Res. 2014;124:31-82.

20. Zagzag D, Lukyanov Y, Lan L, Ali MA, Esencay M, Mendez O, et al. Hypoxia-inducible factor 1 and VEGF upregulate CXCR4 in glioblastoma: implications for angiogenesis and glioma cell invasion. Lab Investig. 2006;86:1221.

21. Sanders VJ, Everall IP, Johnson RW, Masliah E, Group H. Fibroblast growth factor modulates HIV coreceptor CXCR4 expression by neural cells. J Neurosci Res. 2000;59:671-9.

22. Esencay M, Newcomb EW, Zagzag D. HGF upregulates CXCR4 expression in gliomas via NF-kappaB: implications for glioma cell migration. J neuro-Oncol. 2010;99:33-40.

23. Chen P, Cai X, Yang Y, Chen Z, Qiu J, Yu N, et al. Nuclear respiratory factor-1 (NRF-1) regulates transcription of the CXC 
receptor 4 (CXCR4) in the rat retina. Investigative Ophthalmol Vis Sci. 2017;58:4662-9.

24. Moriuchi M, Moriuchi H, Margolis DM, Fauci AS. USF/c-Myc enhances, while Yin-Yang 1 suppresses, the promoter activity of CXCR4, a coreceptor for HIV-1 entry. J Immunol (Baltim, Md: 1950). 1999;162:5986-92.

25. Baron N, Deuster O, Noelker C, Stüer C, Strik H, Schaller C, et al. Role of macrophage migration inhibitory factor in primary glioblastoma multiforme cells. J Neurosci Res. 2011;89:711-7.

26. Lourenco S, Teixeira VH, Kalber T, Jose RJ, Floto RA, Janes SM. Macrophage migration inhibitory factor-CXCR4 is the dominant chemotactic axis in human mesenchymal stem cell recruitment to tumors. J Immunol. 2015;194:3463-74.

27. Bouska A, Zhang W, Gong Q, Iqbal J, Scuto A, Vose J, et al. Combined copy number and mutation analysis identifies oncogenic pathways associated with transformation of follicular lymphoma. Leukemia. 2017;31:83-91.

28. Chen J, Xu-Monette ZY, Deng L, Shen Q, Manyam GC, Martinez-Lopez A, et al. Dysregulated CXCR4 expression promotes lymphoma cell survival and independently predicts disease progression in germinal center B-cell-like diffuse large B-cell lymphoma. Oncotarget. 2015;6:5597-614.

29. Spoo AC, Lübbert M, Wierda WG, Burger JA. CXCR4 is a prognostic marker in acute myelogenous leukemia. Blood. 2007; 109:786-91.

30. Crazzolara R, Kreczy A, Mann G, Heitger A, Eibl G, Fink FM, et al. High expression of the chemokine receptor CXCR4 predicts extramedullary organ infiltration in childhood acute lymphoblastic leukaemia. Br J Haematol. 2001;115:545-53.

31. Kurtova AV, Tamayo AT, Ford RJ, Burger JA. Mantle cell lymphoma cells express high levels of CXCR4, CXCR5, and VLA4 (CD49d): importance for interactions with the stromal microenvironment and specific targeting. Blood. 2009;113:4604-13.

32. Paiva B, Corchete LA, Vidriales MB, Puig N, Maiso P, Rodriguez I, et al. Phenotypic and genomic analysis of multiple myeloma minimal residual disease tumor cells: a new model to understand chemoresistance. Blood. 2016;127:1896-906.

33. Konoplev S, Jorgensen JL, Thomas DA, Lin E, Burger J, Kantarjian HM, et al. Phosphorylated CXCR4 is associated with poor survival in adults with B-acute lymphoblastic leukemia. Cancer. 2011;117:4689-95.

34. Burger JA, Burger M, Kipps TJ. Chronic lymphocytic leukemia B cells express functional CXCR4 chemokine receptors that mediate spontaneous migration beneath bone marrow stromal cells. Blood. 1999;94:3658-67.

35. Ghobrial IM, Bone ND, Stenson MJ, Novak A, Hedin KE, Kay $\mathrm{NE}$, et al. Expression of the chemokine receptors CXCR4 and CCR7 and disease progression in B-cell chronic lymphocytic leukemia/ small lymphocytic lymphoma. Mayo Clin Proc. 2004; 79:318-25.

36. Majid A, Lin TT, Best G, Fishlock K, Hewamana S, Pratt G, et al. CD49d is an independent prognostic marker that is associated with CXCR4 expression in CLL. Leuk Res. 2011;35:750-6.

37. Waldenström J. Incipient myelomatosis or «essential « hyperglobulinemia with fibrinogenopenia-a new syndrome? Acta Med Scandinavica. 1944;117:216-47.

38. Bing J, Fog M, Neel AV. Reports of a third case of hyperglobulinemia with affection of the central nervous system on a toxiinfectious basis. Acta Med Scandinavica. 1937;91:409-27.

39. Bing J, Neel AV. Two cases of hyperglobulinaemia with affection of the central nervous system on a toxi-infectious basis.1. Acta Med Scandinavica. 1936;88:492-506.

40. Buske C, Leblond V, Dimopoulos M, Kimby E, Jager U, Dreyling M, et al. Waldenstrom's macroglobulinaemia: ESMO Clinical Practice Guidelines for diagnosis, treatment and follow-up. Ann Oncol: Off J Eur Soc Med Oncol. 2013;24 (Suppl 6):vi155-9.

41. Owen RG, Treon SP, Al-Katib A, Fonseca R, Greipp PR, McMaster ML, et al. Clinicopathological definition of Waldenstrom's macroglobulinemia: consensus panel recommendations from the Second International Workshop on Waldenstrom's Macroglobulinemia. Semin Oncol. 2003;30:110-5.

42. Treon SP. How I treat Waldenstrom macroglobulinemia. Blood. 2015;126:721-32.

43. Dimopoulos MA, Kastritis E. How I treat Waldenström macroglobulinemia. Blood. 2019;134:2022-35.

44. Buske C, Leblond V, Dimopoulos M, Kimby E, Jäger U, Dreyling M. Waldenström's macroglobulinaemia: ESMO Clinical Practice Guidelines for diagnosis, treatment and follow-up $\dagger$. Ann Oncol. 2013;24:vi155-9.

45. Wang H, Chen Y, Li F, Delasalle K, Wang J, Alexanian R, et al. Temporal and geographic variations of Waldenstrom macroglobulinemia incidence: a large population-based study. Cancer. 2012;118:3793-800.

46. Treon SP, Hunter ZR, Aggarwal A, Ewen EP, Masota S, Lee C, et al. Characterization of familial Waldenstrom's macroglobulinemia. Annals of oncology: official journal of the European Society for. Med Oncol. 2006;17:488-94.

47. Leleu X, Gay J, Roccaro AM, Moreau A-S, Poulain S, Dulery R, et al. Update on therapeutic options in Waldenström macroglobulinemia. Eur J Haematol. 2009;82:1-12.

48. Castillo JJ, Gustine J, Meid K, Dubeau T, Hunter ZR, Treon SP. Histological transformation to diffuse large B-cell lymphoma in patients with Waldenström macroglobulinemia. Am J Hematol. 2016;91:1032-5.

49. Horsman DE, Card RT, Skinnider LF. Waldenstrom macroglobulinemia terminating in acute leukemia: a report of three cases. Am J Hematol. 1983;15:97-101.

50. Castillo JJ, Olszewski AJ, Kanan S, Meid K, Hunter ZR, Treon SP. Overall survival and competing risks of death in patients with Waldenstrom macroglobulinaemia: an analysis of the Surveillance, Epidemiology and End Results database. Br J Haematol. 2015;169:81-9.

51. Castillo JJ, Olszewski AJ, Cronin AM, Hunter ZR, Treon SP. Survival trends in Waldenström macroglobulinemia: an analysis of the Surveillance, Epidemiology and End Results database. Blood. 2014;123:3999-4000.

52. Babwah A, Gustine J, Meid K, Dubeau T, Xu L, Yang G, et al. Long survival in patients with Waldenström macroglobulinaemia diagnosed at a young age. Br J Haematol. 2019;185:799-802.

53. Burnet FM. Immunity, aging and cancer: medical aspects of mutation and selection. San Francisco: Freeman; 1976.

54. Spengler GA, Siebner H, Riva G. Chromosomal abnormalities in macroglobulinemia Waldenstrom: discordant findings in uniovular twins. Acta Med Scandinavica Supplementum. 1966;445:132-9.

55. Goh KO, Swisher SN. Macroglobulinemia of waldenstrom and the chromosomal morphology. Am J Med Sci. 1970;60:237-44.

56. Hanzis C, Ojha RP, Hunter Z, Manning R, Lewicki M, Brodsky $\mathrm{P}$, et al. Associated malignancies in patients with waldenström's macroglobulinemia and their kin. Clin Lymphoma Myeloma Leuk. 2011;11:88-92.

57. Nguyen-Khac F, Lambert J, Chapiro E, Grelier A, Mould S, Barin C, et al. Chromosomal aberrations and their prognostic value in a series of 174 untreated patients with Waldenström's macroglobulinemia. Haematologica. 2013;98:649-54.

58. Hunter ZR, Xu L, Yang G, Zhou Y, Liu X, Cao Y, et al. The genomic landscape of Waldenstrom macroglobulinemia is characterized by highly recurring MYD88 and WHIM-like CXCR4 mutations, and small somatic deletions associated with B-cell lymphomagenesis. Blood. 2014;123:1637-46. 
59. Hunter ZR, Yang G, Xu L, Liu X, Castillo JJ, Treon SP. Genomics, signaling, and treatment of waldenström macroglobulinemia. J Clin Oncol. 2017;35:994-1001.

60. Poulain S, Herbaux C, Bertrand E, Decambron A, Fouquet G, Boyle E, et al. Genomic studies have identified multiple mechanisms of genetic changes in waldenström macroglobulinemia. Clin Lymphoma Myeloma Leuk. 2013;13:202-4.

61. Sacco A, Zhang Y, Maiso P, Manier S, Rossi G, Treon SP, et al. microRNA aberrations in waldenström macroglobulinemia. Clin Lymphoma Myeloma Leuk. 2013;13:205-7.

62. Hunter ZR, Yang G, Xu L, Liu X, Castillo JJ, Treon SP. Genomics, signaling, and treatment of waldenstrom macroglobulinemia. J Clin Oncol. 2017;35:994-1001.

63. Poulain S, Roumier C, Decambron A, Renneville A, Herbaux C, Bertrand E, et al. MYD88 L265P mutation in Waldenstrom macroglobulinemia. Blood. 2013;121:4504-11.

64. Treon SP, Gustine J, Meid K, Yang G, Xu L, Liu X, et al. Ibrutinib monotherapy in symptomatic, treatment-naive patients with waldenstrom macroglobulinemia. J Clin Oncol. 2018;36:2755-61.

65. Rossi D. Role of MYD88 in lymphoplasmacytic lymphoma diagnosis and pathogenesis. Hematol Am Soc Hematol Educ Program. 2014;2014:113-8.

66. Yu X, Li W, Deng Q, Li L, Hsi ED, Young KH, et al. MYD88 L265P mutation in lymphoid malignancies. Cancer Res. 2018; 78:2457-62.

67. Pal Singh S, Dammeijer F, Hendriks RW. Role of Bruton's tyrosine kinase in B cells and malignancies. Mol Cancer. 2018;17:57.

68. Castillo JJ, Xu L, Gustine JN, Keezer A, Meid K, Dubeau TE, et al. CXCR4 mutation subtypes impact response and survival outcomes in patients with Waldenstrom macroglobulinaemia treated with ibrutinib. Br J Haematol. 2019;187:356-63.

69. Treon SP, Xu L, Yang G, Zhou Y, Liu X, Cao Y, et al. MYD88 L265P somatic mutation in Waldenstrom's macroglobulinemia. N. Engl J Med. 2012;367:826-33.

70. Cao Y, Hunter ZR, Liu X, Xu L, Yang G, Chen J, et al. The WHIM-like CXCR4(S338X) somatic mutation activates AKT and ERK, and promotes resistance to ibrutinib and other agents used in the treatment of Waldenstrom's Macroglobulinemia. Leukemia. 2015;29:169-76.

71. Cao Y, Hunter ZR, Liu X, Xu L, Yang G, Chen J, et al. CXCR4 WHIM-like frameshift and nonsense mutations promote ibrutinib resistance but do not supplant MYD88L265P-directed survival signalling in Waldenström macroglobulinaemia cells. Br J Haematol. 2015;168:701-7.

72. Gustine JN, Xu L, Tsakmaklis N, Demos MG, Kofides A, Chen JG, et al. CXCR4 (S338X) clonality is an important determinant of ibrutinib outcomes in patients with Waldenström macroglobulinemia. Blood Adv. 2019;3:2800-3.

73. Xu L, Hunter ZR, Tsakmaklis N, Cao Y, Yang G, Chen J, et al. Clonal architecture of CXCR4 WHIM-like mutations in Waldenström Macroglobulinaemia. Br J Haematol. 2016;172:735-44.

74. Hunter ZR, Xu L, Yang G, Tsakmaklis N, Vos JM, Liu X, et al. Transcriptome sequencing reveals a profile that corresponds to genomic variants in Waldenström macroglobulinemia. Blood. 2016;128:827-38.

75. Varettoni M, Zibellini S, Defrancesco I, Ferretti V, Rizzo E, Malcovati L, et al. Pattern of somatic mutations in patients with Waldenström macroglobulinemia or IgM monoclonal gammopathy of undetermined significance. Haematologica. 2017;102: haematol.2017.172718

76. Treon SP, Tripsas CK, Meid K, Warren D, Varma G, Green R, et al. Ibrutinib in previously treated Waldenstrom's macroglobulinemia. N. Engl J Med. 2015;372:1430-40.

77. Treon SP, Xu L, Hunter Z. MYD88 mutations and response to ibrutinib in Waldenstrom's macroglobulinemia. N. Engl J Med. 2015;373:584-6.
78. Dimopoulos MA, Tedeschi A, Trotman J, Garcia-Sanz R, Macdonald D, Leblond V, et al. Phase 3 trial of ibrutinib plus rituximab in waldenstrom's macroglobulinemia. N. Engl J Med. 2018;378:2399-410.

79. Owen RG, McCarthy H, Rule S, D'Sa S, Thomas SK, Tournilhac $\mathrm{O}$, et al. Acalabrutinib monotherapy in patients with Waldenstrom macroglobulinemia: a single-arm, multicentre, phase 2 study. Lancet Haematol. 2020;7:e112-21.

80. Tam CS, Opat S, D'Sa S, Jurczak W, Lee HP, Cull G, et al. A randomized phase 3 trial of zanubrutinib versus ibrutinib in symptomatic Waldenstrom Macroglobulinemia: the aspen study. Blood. 2020;136:2038-50.

81. Dimopoulos MA, Anagnostopoulos A, Kyrtsonis MC, Castritis E, Bitsaktsis A, Pangalis GA. Treatment of relapsed or refractory Waldenstrom's macroglobulinemia with bortezomib. Haematologica. 2005;90:1655-8.

82. Chen CI, Kouroukis CT, White D, Voralia M, Stadtmauer E, Stewart AK, et al. Bortezomib is active in patients with untreated or relapsed Waldenstrom's macroglobulinemia: a phase II study of the National Cancer Institute of Canada Clinical Trials Group. J Clin Oncol. 2007;25:1570-5.

83. Ghobrial IM, Hong F, Padmanabhan S, Badros A, Rourke M, Leduc R, et al. Phase II trial of weekly bortezomib in combination with rituximab in relapsed or relapsed and refractory Waldenstrom macroglobulinemia. J Clin Oncol. 2010;28:1422-8.

84. Sklavenitis-Pistofidis R, Capelletti M, Liu CJ, Reidy M, Zavidij O, Huynh D, et al. Bortezomib overcomes the negative impact of CXCR4 mutations on survival of Waldenstrom macroglobulinemia patients. Blood. 2018;132:2608-12.

85. Kersten MJ, Minnema M, Amaador K, Vos JM, Nasserinejad K, Kap M, et al. Ixazomib, rituximab and dexamethasone (ird) in patients with relapsed or progressive waldenstrom's macroblobulinemia: final analysis of the HOVON124/ECWM-R2 TRIAL. HemaSphere. 2020;4:S226.

86. Castillo JJ, Meid K, Flynn CA, Chen J, Demos MG, Guerrera ML, et al. Ixazomib, dexamethasone and rituximab in treatment naive Waldenström macroglobulinemia patients: Long-term follow-up Blood advances. Blood Adv. 2020;4:3952-9.

87. Treon SP, Tripsas CK, Meid K, Kanan S, Sheehy P, Chuma S, et al. Carfilzomib, rituximab, and dexamethasone (CaRD) treatment offers a neuropathy-sparing approach for treating Waldenstrom's macroglobulinemia. Blood. 2014;124:503-10.

88. Laribi K, Poulain S, Willems L, Merabet F, Le Calloch R, Eveillard JR, et al. Bendamustine plus rituximab in newlydiagnosed Waldenstrom macroglobulinaemia patients. A study on behalf of the French Innovative Leukaemia Organization (FILO). Br J Haematol. 2018.

89. Burger JA, Peled A. CXCR4 antagonists: targeting the microenvironment in leukemia and other cancers. Leukemia. 2009;23: 43-52.

90. Schepky AG, Bensch KW, Schulz-Knappe P, Forssmann WG. Human hemofiltrate as a source of circulating bioactive peptides: determination of amino acids, peptides and proteins. Biomed Chromatogr: BMC. 1994;8:90-4.

91. Munch J, Standker L, Forssmann W-G, Kirchhoff F. Discovery of modulators of HIV-1 infection from the human peptidome. Nat Rev Micro. 2014;12:715-22.

92. Fiegl M, Samudio I, Clise-Dwyer K, Burks JK, Mnjoyan Z, Andreeff M. CXCR4 expression and biologic activity in acute myeloid leukemia are dependent on oxygen partial pressure. Blood. 2009;113:1504-12.

93. De Clercq E. The bicyclam AMD3100 story. Nat Rev Drug Discov. 2003;2:581.

94. Kashyap MK, Kumar D, Jones H, Amaya-Chanaga CI, Choi MY, Melo-Cardenas J, et al. Ulocuplumab (BMS-936564 / MDX1338): a fully human anti-CXCR4 antibody induces cell death in chronic 
lymphocytic leukemia mediated through a reactive oxygen speciesdependent pathway. Oncotarget. 2016;7:2809-22.

95. Ghobrial IM, Liu CJ, Redd RA, Perez RP, Baz R, Zavidij O, et al. A phase Ib/II trial of the first-in-class anti-CXCR4 antibody ulocuplumab in combination with lenalidomide or bortezomib plus dexamethasone in relapsed multiple myeloma. Clin Cancer Res. 2020;26:344-53.

96. Roccaro AM, Sacco A, Jimenez C, Maiso P, Moschetta M, Mishima Y, et al. C1013G/CXCR4 acts as a driver mutation of tumor progression and modulator of drug resistance in lymphoplasmacytic lymphoma. Blood. 2014;123:4120-31.

97. McDermott DH, Liu Q, Velez D, Lopez L, Anaya-O’Brien S, Ulrick $\mathrm{J}$, et al. A phase 1 clinical trial of long-term, low-dose treatment of WHIM syndrome with the CXCR4 antagonist plerixafor. Blood. 2014;123:2308-16.

98. Skerlj RT, Bridger GJ, Kaller A, McEachern EJ, Crawford JB, Zhou Y, et al. Discovery of novel small molecule orally bioavailable $\mathrm{C}-\mathrm{X}-\mathrm{C}$ chemokine receptor 4 antagonists that are potent inhibitors of T-tropic (X4) HIV-1 replication. J Med Chem. 2010;53:3376-88.

99. Cao Y, Yang G, Hunter ZR, Liu X, Xu L, Chen J, et al. The BCL2 antagonist ABT-199 triggers apoptosis, and augments ibrutinib and idelalisib mediated cytotoxicity in CXCR4 Wildtype and CXCR4 WHIM mutated Waldenstrom macroglobulinaemia cells. Br J Haematol. 2015;170:134-8.

100. Castillo JJ, Gustine J, Meid K, Dubeau T, Keezer A, Allan J, et al. Multicenter prospective phase ii study of venetoclax in patients with previously treated waldenstrom macroglobulinemia. Blood 2018;132:1.
101. Schuster SJ, Bishop MR, Tam CS, Waller EK, Borchmann P, McGuirk JP, et al. Tisagenlecleucel in adult relapsed or refractory diffuse large B-cell lymphoma. N Engl J Med. 2018;380:45-56.

102. Neelapu SS, Locke FL, Bartlett NL, Lekakis LJ, Miklos DB, Jacobson CA, et al. Axicabtagene ciloleucel CAR T-cell therapy in refractory large B-cell lymphoma. N Engl J Med. 2017;377: 2531-44.

103. Wang M, Munoz J, Goy A, Locke FL, Jacobson CA, Hill BT, et al. KTE-X19 CAR T-Cell Therapy in Relapsed or Refractory Mantle-Cell Lymphoma. N Engl J Med. 2020;382:1331-42.

104. Hirayama AV, Gauthier J, Hay KA, Voutsinas JM, Wu Q, Pender BS, et al. High rate of durable complete remission in follicular lymphoma after CD19 CAR-T cell immunotherapy. Blood. 2019;134:636-40.

105. Bansal R, Jurcic JG, Sawas A, Mapara MY, Reshef R. Chimeric antigen receptor $\mathrm{T}$ cells for treatment of transformed Waldenström macroglobulinemia. Leuk Lymphoma. 2020;61:465-8.

106. Jiménez C, Sebastián E, Chillón MC, Giraldo P, Mariano Hernández J, Escalante F, et al. MYD88 L265P is a marker highly characteristic of, but not restricted to, Waldenström's macroglobulinemia. Leukemia. 2013;27:1722-8.

107. Krysiak K, Gomez F, White BS, Matlock M, Miller CA, Trani L, et al. Recurrent somatic mutations affecting B-cell receptor signaling pathway genes in follicular lymphoma. Blood. 2017;129: 473-83.

108. Dimopoulos MA, Tedeschi A, Trotman J, García-Sanz R, Macdonald D, Leblond V, et al. Phase 3 trial of ibrutinib plus rituximab in Waldenström's macroglobulinemia. N Engl J Med. 2018;378:2399-410. 